\title{
Análisis estructural de la web académica iberoamericana
}

\author{
José Luis Ortega*, Isidro F. Aguillo*
}

Resumen: El objetivo de este trabajo es presentar de una forma visual la red de dominios web universitarios más importantes de Iberoamérica. En él se muestra las características topológicas y se describe las relaciones web entre universidades de diferentes países y continentes. Se seleccionaron las primeras 350 instituciones iberoamericanas de educación superior del Ranking Web of World Universities y se extrajeron sus enlaces a partir de Yahoo! Search. Se construyeron con estos datos mapas geográficos y gráficos de redes. Se utilizaron técnicas de Análisis de Redes Sociales para analizar y describir las propiedades estructurales de toda la red y sus nodos. Los resultados muestran que la red iberoamericana está constituida por subredes nacionales que se fusionan en un núcleo central. La subred española domina la red iberoamericana, la cual está dividida en dos regiones lingüísticas: portugués (Brasil y Portugal) y español (los restantes países hispanoamericanos).

Palabrasclave: visualización de información, análisis de redes sociales, cibermetría, web universitaria.

\section{Structural analysis of the Iberoamerican academic web}

Abstract:This work provides a visual display of the domain network the most important Iberoamerican universities. The topological characteristics of this network are described, and the web relationships among the universities of different countries and continents are examined. The top 350 institutions of higher education of Iberoamerica were selected from the Ranking Web of World Universities and their link relationships obtained from Yahoo! Search. Network graphs and geographical maps were constructed from the search engine data. Social network analysis techniques were used to analyse and describe the structural properties of the network and its nodes. The results show that the Iberoamerican network is composed of national sub-networks that merge to form a central core. The Spanish language sub-network dominates the Iberoamerican network, which is split into two linguistic regions: Portuguese (Brazil and Portugal) and Spanish (the remaining countries).

Keywords: information visualization, social network analysis, webometrics, university web.

* Laboratorio de Cibermetría, CCHS-CSIC, Madrid, España. Correo-e: isidro.aguillo@cchs.csic.es; jortega@orgc.csic.es.

Recibido: 3-11-08; 2. ${ }^{\mathrm{a}}$ versión: 2-3-09. 


\section{Introducción}

La web está siendo un motor de cambio en la evolución de las universidades. Esta ha permitido el desarrollo de nuevos modelos de docencia como la educación a distancia y la formación no presencial, que está posibilitando un desarrollo de la universidad más allá de su ámbito local y geográfico. Las universidades extienden así su capacidad formativa a nuevos segmentos de la población y atraen alumnos de lugares distantes salvando limitaciones geográficas (Ryan et al., 2000). Si tenemos en cuenta la dimensión investigadora, la web ha ampliado la difusión de resultados científicos a través de nuevos medios (paginas personales, repositorios institucionales, etc.) que permiten mostrar la capacidad investigadora de estas instituciones, siendo una muestra de prestigio que les permite atraer recursos económicos y humanos de gran valor (Bates, 2001). Esta nueva realidad está incrementando la competitividad entre las universidades que pugnan por atraer más alumnos y recursos, lo que ha llevado consigo un intento de evaluar la actividad y el desarrollo de las universidades en este medio paralelo de la web. La Cibermetría, como disciplina, se ha centrado en este aspecto con la intención de explicar y describir cómo es la situación académica en la web y cómo es posible evaluar el esfuerzo científico y educativo que estas instituciones están llevando a cabo en la red (Thelwall, 2004).

\section{Estudios relacionados}

La actividad y la producción en la web ha sido usada como un indicador de la actividad educativa y científica, relacionando indicadores web con resultados académicos (Thelwall, 2002a; Thelwall y Harries, 2003; 2004; Smith, 2008) o con indicadores bibliométricos (Aguillo, Granadino, Ortega y Prieto, 2006). La visualización de información (Chen, 2003) también ha sido una idónea herramienta para representar relaciones entre universidades y mostrar visualmente cómo determinadas variables afectan a estas relaciones. En un primer momento se usó análisis multivariante para representar y agrupar universidades (Polanco, Boudourides, Besagni y Roche, 2001; Vaughan, 2006) en función de su patrón de enlaces. Ahora, el Análisis de Redes ofrece posibilidades visuales y estructurales adicionales. Así, Heimeriks y Van den Besselaar (2006) usaron estas técnicas de análisis para identificar cuatro zonas geográficas en el espacio web de la Unión Europea (UE): Escandinavia, Reino Unido, Alemania y Sur de Europa. Ortega et al. (2008) también obtuvieron resultados similares, descubriendo que las universidades europeas se agrupan en subredes nacionales o locales las cuales se conectan con las restantes redes en función de criterios lingüísticos y geográficos (Thelwall, 2002b; Thelwall, Tang y Price, 2003). Últimamente, Thelwall y Zuccala (2008) estudiaron la relación entre los enlaces de universidades y espacios web nacionales en Europa, describiendo dichas relaciones al nivel de países. 
Muchos estudios se han centrado en el desarrollo web a nivel de países. Así en España encontramos los trabajos de Thelwall y Aguillo (2003) y Ortega y Aguillo (2007), en Canadá los de Vaughan y Thelwall (2005) y Vaughan (2006) o análisis supranacionales como los de la UE (Heimeriks y van den Besselaar, 2006; Ortega et al., 2008; Thelwall y Zuccala, 2008) o Escandinavia (Ortega y Aguillo, 2008).

En el mundo iberoamericano cabe destacar las caracterizaciones hechas de la web española y chilena por Baeza-Yates, Castillo y Lopez (2005) y Baeza-Yates, Castillo y Graells (2006) y las realizadas sobre Sudamérica (Bordignon y Tolosa, 2006), Paraguay (Bordignon et al., 2006) y Argentina (Tolosa et al., 2007). Aguillo, Granadino y Llamas (2005) realizaron una primera aproximación a la web académica iberoamericana desde criterios de diseño y posicionamiento, mientras Caraballo-Pérez et al. (2008) describieron la web académica cubana desde estos mismos criterios. Aguillo (2005) y Aguillo et al. (2007) definieron indicadores de contenido para la evaluación de este espacio web. Aparte de estudios descriptivos y analíticos, también se han publicado diversos trabajos teóricos sobre el ámbito de la cibermetría (Vanti, 2005).

\section{Objectivos}

El objetivo de este trabajo es presentar de una forma visual las 350 universidades más importantes en Iberoamérica de acuerdo al Ranking Web of World Universities (www.webometrics.info). Este mapa pretende mostrar características topológicas de esta red y describir las relaciones entre universidades de diferentes países y continentes. También se muestra, a través de técnicas de análisis de redes, las universidades más destacadas en la estructura de la red, las universidades pasarela que conectan diferentes espacios web o subredes y el núcleo más conectado de la red.

\section{Metodología}

\subsection{Datos}

Se seleccionaron las primeras 350 universidades iberoamericanas listadas en el World Universities Ranking on the Web (webometrics.info). Este número fue considerado representativo ya que permitía mostrar de una forma amplia y representativa la mayoría de países iberoamericanos. Dicho ranking ordena más de 10.000 universidades del mundo en función de cuatro criterios básicos: Número de páginas, que muestran la producción de contenidos de estas universidades en la web; número de enlaces entrantes, que muestran la visibilidad de dichos dominios web; número de ficheros ricos (ppt, pdf, etc.), los cuales informan de la 
actividad académica y científica de cada universidad y registros en Google Scholar, lo que indica la producción científica accesible vía web.

Aparte de los criterios cibermétricos utilizados para valorar estas universidades en la web, este ranking es el listado más completo de universidades en la web, por lo que podemos considerarla como la fuente más adecuada para estudios sobre el desarrollo académico en la web.

Una vez seleccionadas las 350 primeras universidades iberoamericanas se procedió a obtener una matriz de enlaces entre estas universidades. Esta matriz es de $350 \times 350$ de modo no simétrico, ya que los enlaces que parten de una universidad A hacia una universidad B no tienen porqué ser los mismos que de la universidad B van hacia la universidad A. Para ello, se realizaron de forma automática 122.500 consultas al buscador Yahoo! Search. Este buscador fue seleccionado porque es el único, de gran cobertura, que permite el uso combinado de operadores. La fecha de toma de datos fue agosto de 2008. Las consultas utilizadas fueron:

\begin{tabular}{|l|l|}
\hline $\begin{array}{l}\text { site: }\{\text { universidad } \mathrm{A}\} \text { linkdomain: \{uni- } \\
\text { versidad } \mathrm{B}\}\end{array}$ & $\begin{array}{l}\text { Número de enlaces que apuntan a la universidad B } \\
\text { provenientes de páginas de la universidad A. }\end{array}$ \\
\hline Site: $\{$ universidad $\mathrm{A}\}$ & Volumen total de páginas de cada universidad. \\
\hline
\end{tabular}

\subsection{Construcción de las redes}

Una vez obtenida la matriz, esta se adaptó y procesó por el programa de análisis de redes Pajek 1.02 (Nooy, Mrvar y Batagelj, 2005). Con dicho programa se obtuvo la red de universidades iberoamericanas. Además de la red, se le añadieron una serie de atributos que permitieran explicar de forma visual los resultados obtenidos. Así el tamaño de cada nodo representa el volumen total de páginas indizadas en Yaboo! Search y el color la nacionalidad de cada universidad. Para una mejor visualización del gráfico y una significación mayor de los vínculos se estableció un punto de corte de más de 5 enlaces.

El análisis de la red se realizó a través de medidas de Análisis de Redes Sociales (ARS). Estas nos permiten conocer las principales características estructurales de la red como puede ser su tipología, su diámetro y su densidad además de conocer qué subunidades la forman. Por otro lado, estas medidas nos permiten conocer la función e importancia que poseen determinados nodos dentro de la red. Las medidas usadas son:

- K-Cores: se define como una subred en la que cada nodo tiene al menos un grado $k$. K-Cores detecta grupos con una fuerte densidad de enlaces. En redes de escala libre como la web el núcleo con el grado más alto es el núcleo central, detectando el grupo de nodos donde descansa la red (Seidman, 1983). 
- Grado (k): el número de líneas que conectan a un nodo. Estas pueden ser normalizadas (Grado normalizado) por el número total de nodos en la red. En una red dirigida como la web se puede computar los enlaces entrantes (Grado entrante) y los enlaces salientes (Grado saliente). En Cibermetría, el Grado entrante se ha señalado como indicador de la visibilidad de un dominio web (Cothey, 2005; Kretschmer \& Kretschmer, 2006), mientras que el Grado saliente es considerado como indicador de generación de tráfico.

- Grado de intermediación: se define como la capacidad de un nodo de mantener conectados aquellos nodos que no están directamente conectados entre sí. Mide el número de veces en el que un nodo aparece en las rutas existentes en la red. Desde un punto de vista cibermétrico, esta medida permite detectar pasarelas que conectan diferentes tramos de la red (Ingwersen, 1998).

- Distancia: es el número mínimo de nodos que median entre otros dos, el promedio de todas las rutas más cortas se denomina la distancia media. Esta medida permite conocer la cohesión de la red, así si esta es corta existe una fuerte cohesión ya que para llegar a cualquier nodo necesitamos pocos intermediarios (Broder et al., 2000).

- Diámetro: es el número de enlaces que distan entre los nodos más alejados entre sí. Al igual que la distancia, esta medida permite medir la cohesión de la red. El diámetro es también es usado para detectar redes de mundo pequeño (Björneborn, 2001).

\subsection{Mapa geográfico}

El mapa geográfico se construyó a partir de los datos obtenidos previamente para el gráfico 2. Estos datos fueron agregados a nivel de país, para mostrar el desarrollo académico de cada país en la web. Para la construcción de dicho mapa se utilizó un sistema de información geográfico (SIG) MapViewer 6 (2005). Para la construcción del mapa se utilizaron dos capas diferentes. La primera representa un mapa de zonas coloreadas gradualmente según el número de páginas que posee cada país. El segundo muestra un mapa de flujos donde se muestra, a través del color y el grosor de las líneas, el número de enlaces que van de un país a otro. Los enlaces y páginas de ambas capas han sido clasificados de acuerdo al método de ruptura natural de Jenks (Jenks, 1963). Este método determina la mejor clasificación de los valores en clases a través de la comparación iterativa de la suma de sus cuadrados entre los valores observados dentro de cada clase y la media de dicha clase. Este algoritmo mejora la visualización al crear diferencias más significativas entre las clases. 


\section{Resultados}

\subsection{Análisis descriptivo}

Antes del análisis de enlaces se ha realizado una distribución del número de universidades por cada país.

TABLA I

Distribución de las 350 universidades por países

\begin{tabular}{|c|c|c|c|c|c|}
\hline País & Dominio & Universidades & $\%$ & Páginas web & $\%$ \\
\hline Brasil & br & 82 & 23,43 & 7.928 .337 & 17,03 \\
\hline España & es & 65 & 18,57 & 22.747 .338 & 48,87 \\
\hline México & $\mathrm{mx}$ & 42 & 12,00 & 4.416 .756 & 9,49 \\
\hline Portugal & $\mathrm{pt}$ & 35 & 10,00 & 4.719 .443 & 10,14 \\
\hline Chile & $\mathrm{cl}$ & 32 & 9,14 & 1.735 .217 & 3,73 \\
\hline Argentina & ar & 31 & 8,86 & 1.617 .898 & 3,48 \\
\hline Colombia & $\mathrm{CO}$ & 19 & 5,43 & 1.441 .534 & 3,10 \\
\hline Puerto Rico & $\mathrm{pr}$ & 9 & 2,57 & 382.999 & 0,82 \\
\hline Perú & pe & 9 & 2,57 & 692.106 & 1,49 \\
\hline Venezuela & ve & 8 & 2,29 & 392.127 & 0,84 \\
\hline Costa Rica & $\mathrm{cr}$ & 5 & 1,43 & 226.709 & 0,49 \\
\hline Ecuador & $\mathrm{ec}$ & 4 & 1,14 & 78.143 & 0,17 \\
\hline Uruguay & uy & 2 & 0,57 & 32.566 & 0,07 \\
\hline El Salvador & SV & 2 & 0,57 & 27.660 & 0,06 \\
\hline Guatemala & gt & 2 & 0,57 & 44.887 & 0,10 \\
\hline Paraguay & py & 1 & 0,29 & 7.155 & 0,02 \\
\hline Cuba & $\mathrm{cu}$ & 1 & 0,29 & 27.980 & 0,06 \\
\hline Bolivia & bo & 1 & 0,29 & 28.816 & 0,06 \\
\hline TOTAL & & 350 & 100,00 & 46.547 .671 & 100,00 \\
\hline
\end{tabular}

La Tabla I muestra la distribución de universidades por sus países. En la muestra se observa que Brasil $(23,43 \%)$ es el país que más universidades o instituciones de educación superior aporta junto España $(18,57 \%)$ y México (12\%). En el lado opuesto están Paraguay, Cuba y Bolivia las que menos aportan a la muestra con tan sólo una universidad. Esta distribución encaja con otras realizadas según criterios bibliométricos (Grupo SCImago, 2007). Sin embargo, si ob- 
servamos el porcentaje de páginas que aporta cada país se aprecia que España es el país con mayor número de páginas $(48,87 \%)$ casi triplicando el número de Brasil (17,03\%), el segundo país en número de páginas. Seguidamente está Portugal $(10,14 \%)$ y México $(9,49 \%)$.

\section{FIGURA 1}

\section{Red de enlaces a nivel de países}

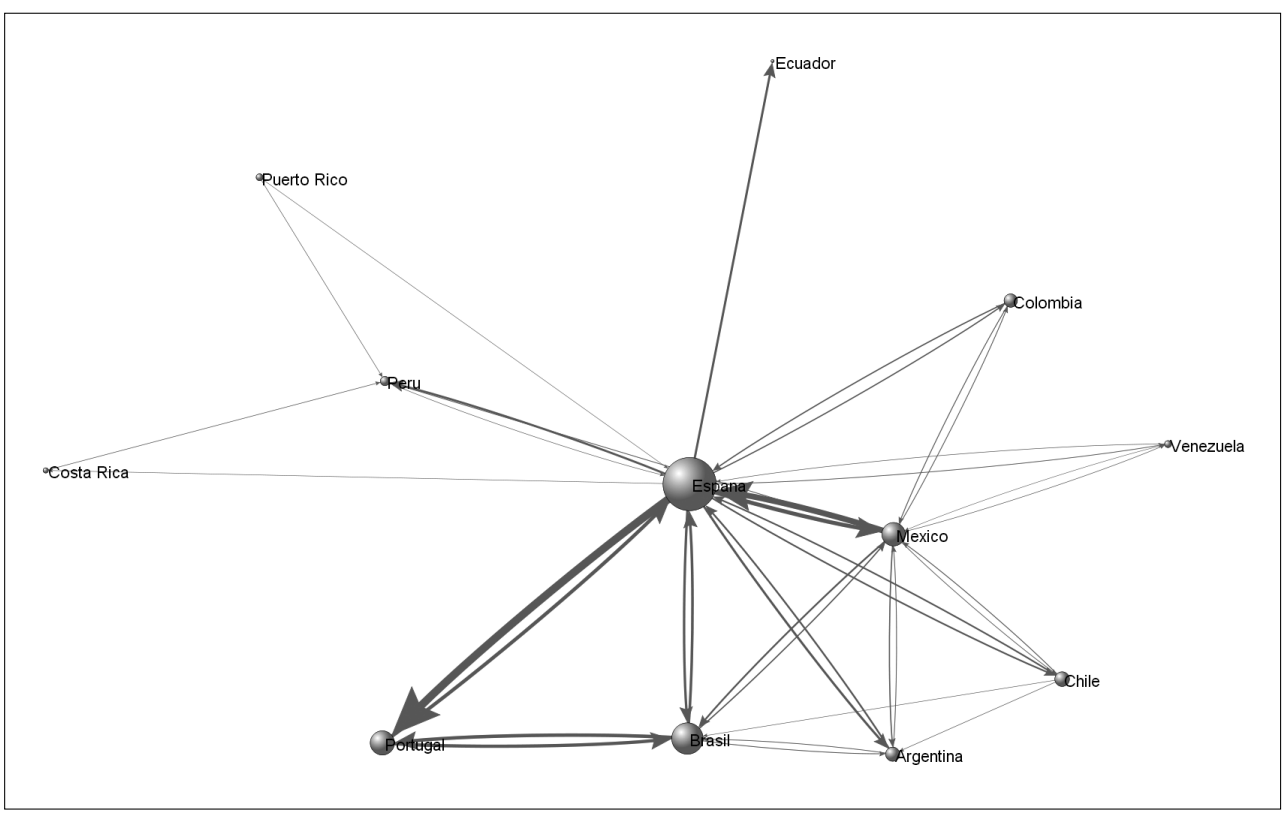

La Figura $1^{1}$ está construida a partir de la agregación de los dominios universitarios a cada país al igual que el número de enlaces. El tamaño de cada nodo muestra el número total de páginas web que cada país posee. Se ha utilizado un punto de corte por encima del 5\% del total de enlaces. En dicha Figura 1 se aprecia la posición central que adquiere España $(k=0,81)$ que junto con México $(k=0,54)$ es el país más conectado con los restantes países. Por el grosor de los enlaces España posee fuertes vínculos con México y Portugal. De esta forma se puede desprender que la red iberoamericana descansa sobre la red española ya que por un lado articula la red hispanoamericana a través de México y por otro lado los países luso-parlantes a través de Portugal.

${ }^{1}$ Las figuras pueden verse a una mayor resolución y en color en la dirección: http://internetlab. cindoc.csic.es/cv/11/iberoamerica. 


\subsection{Mapa geográfico}

En la Figura 2 se aprecian resultados parecidos a la Figura 1. Los países más importantes, tanto por el número de páginas como por el tráfico de enlaces, son España (48,87\%, 22.747 miles de páginas), Brasil (17,03\%, 7.928 miles de páginas) y Portugal (10,14\%, 4.719 miles de páginas), seguidos por México (9,49\%, 4.416 miles de páginas) Chile (3,73\%, 1.735 miles de páginas), Argentina (3,48\%, 1.617 miles de páginas) y Colombia (3,1\%, 1.441 miles de páginas).

En el lado contrario destacan por su escasa presencia en la web los países de Centro América (con la excepción de Costa Rica y Guatemala) y las islas del Caribe (con la excepción de Cuba y Puerto Rico).

El flujo de enlaces muestra un fuerte tráfico entre los países europeos (España y Portugal) con los países americanos, mostrándose aquí también la dualidad lingüística entre hispanohablantes y lusoparlantes. Teniendo en cuenta los flujos interamericanos, destacamos la relación México-Brasil y México-Argentina.

\section{FIGURA 2}

\section{Mapa geográfico de Iberoamérica}

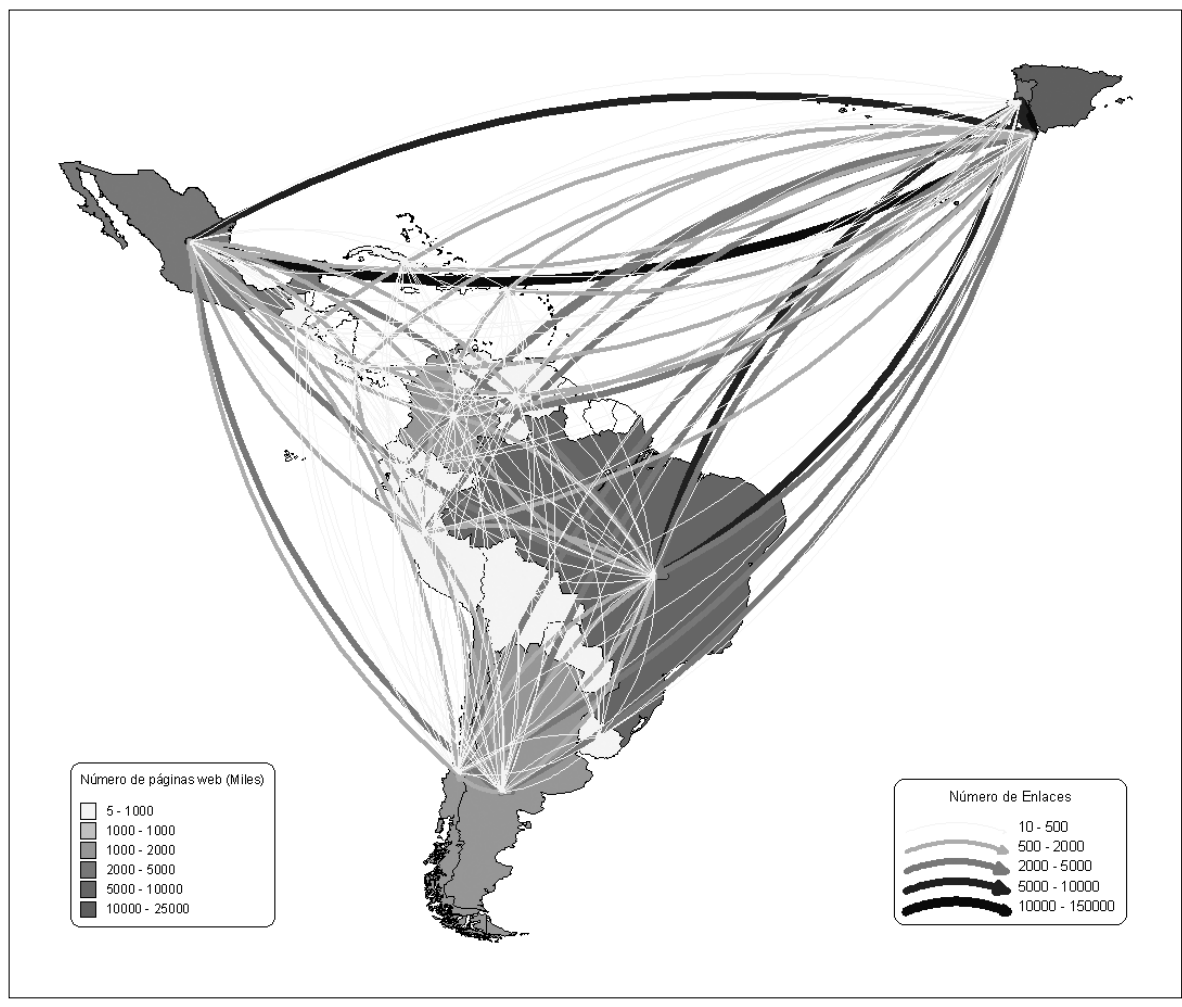




\subsection{Red universitaria}

La Figura 3 muestra la red de las 350 universidades iberoamericanas más importantes en la web. Esta red se ha energizado a través del algoritmo de Fruchterman-Reingold (1991). Este algoritmo actúa como un mecanismo de repulsión alejando los nodos más aislados y acercando los más conectados. Esta característica le permite visualizar de forma más clara las subredes que componen la red. La red posee un alto grado de cohesión ya que su diámetro es de 4 clics, detectándose una distancia media de 2,13 clics. Sin embargo, su cohesión no es mayor que la europea (Ortega et al, 2008), quizás debido a las diferencias lingüísticas y geográficas.

Como se aprecia claramente las universidades se agrupan por sus respectivos países, así se aprecia el grupo de universidades portuguesas, brasileñas, españolas y mexicanas. Además, existe un criterio lingüístico y cultural que relaciona los grupos entre sí. Así se aprecia dos ejes que conforman la red: el eje portugués, constituido por Brasil y Portugal y el eje español, formado por el resto de

\section{FIGURA 3}

\section{Red de los 350 dominios iberoamericanos en la web}

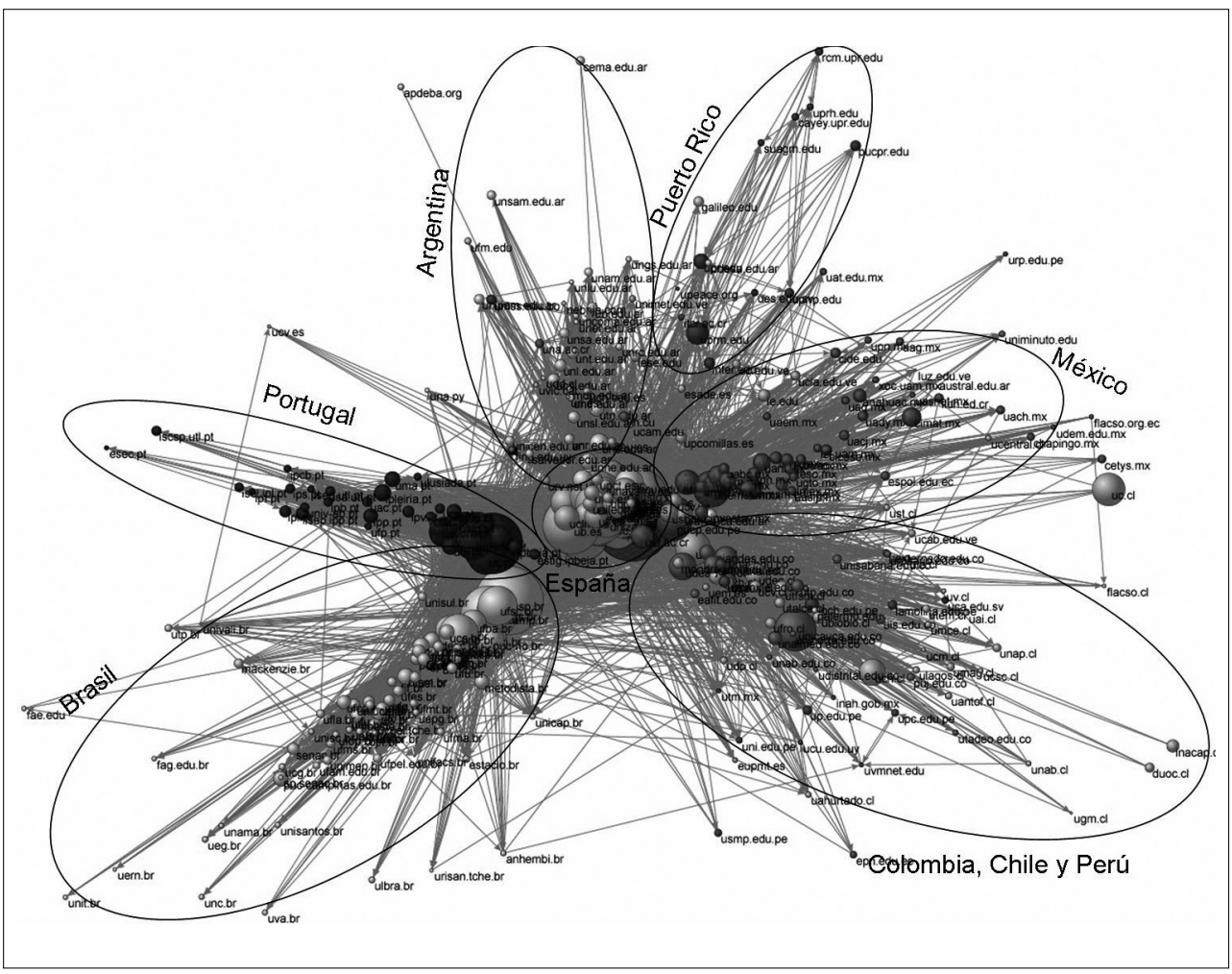


países iberoamericanos. Al igual que las Figuras 1 y 2, se aprecia la posición central de las universidades españolas, las cuales articulan la subred hispanoamericana y la subred luso-americana. Esta afirmación es corroborada al aplicar la técnica de $k$-cores, a través de la cual podemos detectar el núcleo más denso y conectado de la red. Esta técnica ha identificado a un grupo de 70 universidades conectadas entre sí con un grado 47. De entre ellas 50 (71\%) son españolas.

Por otro lado se aprecia que las universidades de mayor tamaño poseen una posición más central, ya que existe una fuerte relación entre el número de páginas y los enlaces entrantes por cada dominio web.

El gráfico muestra también decisiones erróneas que afectan seriamente a la situación de una universidad en la web. Este es el caso de la Pontificia Universidad Católica de Chile (uc.cl). Recientemente cambió su dominio de upc.cl a uc.cl esto le ha causado perder gran parte de los enlaces que recibía. Por este motivo, podemos apreciar claramente como dicha universidad se encuentra muy alejada del centro a pesar del volumen considerable de páginas. Este tipo de decisiones genera una gran cantidad de enlaces rotos que afectan considerablemente a la pérdida de tráfico hacia dichos dominios web.

En la Tabla II se ordenan las universidades más importantes por su grado de centralidad en la red. Estas son la Universidad Nacional Autónoma de México (unam.mx) en México $(k=0,618)$, la Universidad de Sao Paulo (usp.br) en Brasil $(k=0,485)$ y la Universidad de Granada (ugr.es) en España $(k=0,444)$. Como se puede apreciar de las 10 primeras universidad por su grado de centralidad 5 son españolas, 3 brasileñas y 2 mexicanas. Esto confirma la observación anterior de que la red española es el centro de la red, secundada por México y Brasil.

TABLA II

Universidades por su grado de centralidad

\begin{tabular}{l|l|l|l|l|l}
\hline \multicolumn{1}{c|}{ País } & \multicolumn{1}{|c|}{ Universidad } & \multicolumn{1}{c|}{$\begin{array}{c}\text { Dominio } \\
\text { web }\end{array}$} & Grado & $\begin{array}{c}\text { Grado } \\
\text { entrante }\end{array}$ & $\begin{array}{c}\text { Grado } \\
\text { saliente }\end{array}$ \\
\hline MX & U. Nacional Autónoma de México & unam.mx & 0,618 & 0,830 & 0,555 \\
\hline BR & U. de Sao Paulo & usp.br & 0,485 & 0,808 & 0,507 \\
\hline ES & U. de Granada & ugr.es & 0,444 & 0,833 & 0,366 \\
\hline ES & U. Complutense de Madrid & ucm.es & 0,386 & 0,667 & 0,467 \\
\hline BR & U. Federal de Santa Catarina & ufsc.br & 0,368 & 0,805 & 0,335 \\
\hline BR & U. Federal do Rio Grande do Sul & ufrgs.br & 0,365 & 0,696 & 0,363 \\
\hline ES & U. Politécnica de Madrid & upm.es & 0,356 & 0,664 & 0,346 \\
\hline ES & U. de Zaragoza & unizar.es & 0,332 & 0,756 & 0,312 \\
\hline ES & U. de Barcelona & ub.es & 0,330 & 0,693 & 0,309 \\
\hline MX & U. de Guadalajara & udg.mx & 0,262 & 0,790 & 0,232 \\
\hline
\end{tabular}


También es llamativo que aunque España es la subred con mayor centralidad, sea la UNAM de México la que posee mayor grado de centralidad. Este hecho ha sido también observado por Ortega y Aguillo (2009) con respecto a la red mundial. Con lo que podemos afirmar que la principal universidad en la web del ámbito hispano parlante es la UNAM.

Como se aprecia en la Figura 3, cada universidad está agrupada con las universidades de su propio país, detectándose subredes nacionales que convergen en el centro con las restantes subredes de cada país. El grado de intermediación nos permite conocer qué universidades median entre sus propias subredes locales y el conjunto de toda la red. La Tabla III nos muestra la principal universidad de cada uno de los 10 primeros países. Como en la Tabla II, la UNAM vuelve a ser la universidad con mayor intermediación, siendo la universidad que más universidades mantiene conectadas a la red, concretamente un 15\%. El resto de universidades destacan dentro de su país como es la U. de Sao Paulo en Brasil, la U. de Chile en Chile y la U. de Buenos Aires en Argentina. Por lo que podemos considerar que estas universidades actúan de puente entre sus redes locales y la red iberoamericana.

TABLA III

Primeras universidades de cada país por su grado de intermediación

\begin{tabular}{l|l|l|l}
\hline \multicolumn{1}{c|}{ País } & \multicolumn{1}{|c|}{ Universidad } & \multicolumn{1}{c}{ Dominio web } & \multicolumn{1}{c}{$\begin{array}{c}\text { Grado de } \\
\text { intermediación }\end{array}$} \\
\hline MX & U. Nacional Autónoma de México & unam.mx & 0,1509 \\
\hline BR & U. de Sao Paulo & usp.br & 0,0751 \\
\hline CL & U de Chile & uchile.cl & 0,0416 \\
\hline ES & U. de Granada & ugr.es & 0,0344 \\
\hline AR & U. de Buenos Aires & uba.ar & 0,0254 \\
\hline PT & U de Coimbra & uc.pt & 0,0188 \\
\hline PE & Pontificia U. Católica del Perú & pucp.edu.pe & 0,0092 \\
\hline CO & Pontificia U. Javeriana de Bogotá & javeriana.edu.co & 0,0082 \\
\hline CR & U. de Costa Rica & ucr.ac.cr & 0,0052 \\
\hline EC & U. Técnica Particular de Loja & utpl.edu.ec & 0,0038 \\
\hline
\end{tabular}

\section{Discusión}

Los resultados obtenidos permiten ponerlos en contexto con análisis precedentes relacionados con la web académica en distintos ámbitos. Al igual que Heimeriks y Van den Besselaar (2006), Ortega et al (2008) y Ortega y Aguillo (2008), la web académica iberoamericana se estructura en subredes nacionales 
que agrupan las universidades que pertenecen a un mismo país. Sin embargo, por las características propias de la red iberoamericana, emerge un factor lingüístico (Thelwall, Tang y Price, 2003) que ayuda a comprender la configuración de la red. Así se aprecia dos zonas diferenciadas: la hispano-parlante y la luso-parlante. Este patrón también ha sido detectado en el análisis de la web académica canadiense (Vaughan, 2006) y europea (Ortega et al, 2008). Por otro lado, la posición central de las universidades españolas en la red iberoamericana contrasta con su posición periférica en la red europea (Ortega y Aguillo, 2007), lo que nos sugiere que la red española está más volcada hacia el mundo iberoamericano a la vez que nos informa del peso que el factor lingüístico puede poseer a la hora de la configuración de los espacios académicos en la web.

\section{Conclusiones}

La red iberoamericana de universidades en la web está constituida por subredes nacionales donde cada universidad se agrupa con las de su propio país. De esta forma, existe un factor geográfico que explica la agrupación de universidades en subredes nacionales. Dichas subredes se relacionan entre sí de acuerdo a dos ejes lingüísticos que caracterizan a la web académica Iberoamericana. Por un lado, el eje luso-parlante representado por Brasil y Portugal y por otro el eje hispanohablante representado por el resto de países iberoamericanos. El conjunto de la red descansa sobre la red española ya que el $71 \%$ de las universidades agrupadas en el núcleo más conectado de la red son españolas. De esta forma la red española actúa de vínculo entre las dos subgrupos lingüísticos. Por un lado articula la red en portugués a través de sus fuertes vínculos con la red portuguesa y por otro relaciona la red hispana a través de México. Pese a esto la UNAM es la principal universidad en la red ya que posee el mayor grado de centralidad y de intermediación en la red.

\section{Bibliografia}

Aguillo, I. F. (2005): Indicadores de contenidos para la web académica iberoamericana. BiD: textos universitaris de biblioteconomia $i$ documentació, desembre, (15). http:// www2.ub.edu/bid/consulta articulos.php?fichero=15aguil2.htm [Consulta: 29-10-2008].

Aguillo, I. F.; Granadino, B., y Llamas, G. (2005): Posicionamiento en el web del sector académico iberoamericano. Interciencia, vol. 30 (12), 735-738.

Aguillo, I. F.; Granadino, B.; Ortega, J. L.. y Prieto, J. A. (2006): Scientific Research Activity and Communication Measured With Cybermetrics Indicators. Journal of the American Society for Information Science and Technology, vol. 57 (10), 1296-1302.

Aguillo, I. F.; Ortega, J. L.; Prieto, J. A., y Granadino, B. (2007): Indicadores web de actividad científica formal e informal en Latinoamérica. Revista española de Documentación Científica, vol. 30 (1), 49-60. 
Baeza-Yates, R.; Castillo, C., y Lopez, V. (2005): Characteristics of the Web of Spain. Cybermetrics, vol. 9 (1), paper 3 http://www.cindoc.csic.es/cybermetrics/articles/v9i1p3. html [Consulta: 29-10-2008].

Baeza-Yates, R.; Castillo, C., y Graells, E. (2006): Características de la Web Chilena 2006. Universidad de Chile: Santiago http://www.ciw.cl/material/web chilena 2006/index. html [Consulta: 29-10-2008].

Bates, A. (2001): Cómo gestionar el cambio tecnológico: Estrategias para los responsables de centros universitarios. Barcelona, Spain; Gedisa/Universitat Oberta de Catalunya

Björneborn, L. (2001): Small-world linkage and co-linkage. En Proceedings of the 12th ACM conference on hypertext and hypermedia. New York: ACM Press, pp. 133-134.

Bordignon, F. R. A.; Lavallen, P. J., y Tolosa, G. H. (2006): El Estado de la Web de Paraguay y la Sociedad de la Información, En Proceedings of the I Congreso Internacional y VI Congreso Nacional de Bibliotecarios, Documentalistas y Archivistas del Paraguay, Asunción, Paraguay.

Bordignon, F. R. A., y Tolosa, G. H. (2006): Characterization of South American Educational Web Domains, En Proceedings Congreso Argentino de Ciencias de la Computación. CACIC 2006, Potrero de los Funes, Argentina.

Broder, A.; Kumar, R.; Maghoul, F.; Raghavan, P., y Stata, R. (2000): Graph structure in the Web. En Proceedings of the 9th International World Wide Web Conference, Elsevier; Amsterdam.

Caraballo Pérez, Y.; Torres Cárdenas, V.; Noda Amorós, A. C., y Herrera Toscano, J. A. (2008): Medidas de popularidad de la educación superior cubana: un análisis webmétrico. El Profesional de la Información, vol. 17 (4).

Chen, C. (2003): Mapping Scientific Frontiers: The Quest for Knowledge Visualization. London: Springer-Verlag, p. 256.

Cothey, V. (2005): Some preliminary results from a link-crawl of the European Union Research Area Web. En Ingwersen, P; Larsen, B. (editores) Proceeding of the 10th International Conference of the International Society for Scientometrics and Informetrics. Karolinska University Press; Stockholm.

Fruchtermann, T. M. J., y Reingold, E. M. (1991): Graph Drawing by Force-Directed Placement. Software: Practice and Experience, 21 (11), 1129-1164.

Grupo SCImago (2007): Ranking de instituciones de investigación iberoamericanas (RI3). El Profesional de la Información, 16 (3), 258-260.

Heimeriks, G., y Van den Besselaar, P. (2006): Analyzing hyperlinks networks: The meaning of hyperlink based indicators of knowledge production. Cybermetrics, $10(1,1)$. http://www.cindoc.csic.es/cybermetrics/articles/v10i1p1.html [Consulta: 29-10-2008].

Ingwersen, P. (1998): The Calculation of Web Impact Factors. Journal of Documentation, vol. 54 (2), 236-243.

Jenks, G. F. (1963): Generalization in statistical mapping. Annals of the Association of American Geographers, vol. 53, 15-26.

Kretschmer, H., y Kretschmer, T. (2006): Application of a New Centrality Measure for Social Network Analysis to Bibliometric and Webometric Data. Proceeding of the IEEE International Conference on Digital Information Management (ICDIM). IEEE; Bangalore, India. 
MapViewer [cd-rom]. Ver. 6.01.23 [Estados Unidos]: Golden Software, Inc., c1993-2005. Programa informático.

Nooy, W. de; Mrvar, A., y Batagelj, V. (2005): Exploratory Social Network Analysis with Pajek. Cambridge University Press; Cambridge, UK, p. 362.

Ortega, J. L., y Aguillo, I. F. (2007): La Web académica española en el contexto del Espacio Europeo de Educación Superior: Estudio exploratorio. El profesional de la información, vol. 16 (5), 417-425.

Ortega, J. L., y Aguillo, I. F. (2008): Visualization of the Nordic academic web: Link analysis using social network tools. Information Processing \& Management, vol. 44 (4), 1624-1633.

Ortega, J. L., y Aguillo, I. F. (2009): Mapping World-class universities on the Web. Information Processing \& Management, 45(2), 272-275.

Ortega, J. L.; Aguillo, I. F.; Cothey, V., y Scharnhorst, A. (2008): Maps of the academic web in the European Higher Education Area - an exploration of visual web indicators. Scientometrics, vol. 74 (2), 295-308.

Polanco, X.; Boudourides, M.; Besagni, D., y Roche, I. (2001): Clustering and Mapping European University Web Sites Sample for Displaying Associations and Visualizing Networks. Proceeding of the NTTSEETK 2001 Conference. Hersonissos, Crete.

Ryan, S.; Scott, B.; Freeman, H., y Patel, D. (2000): The Virtual University - the Internet and Resource Based Learning. Kogan Page; London, UK, p. 192.

Seidman, S. B. (1983): Network structure and minimum degree. Social Networks, vol. 5, 269-287.

Smith, A. G. (2008): Benchmarking Google Scholar with the New Zealand PBRF research assessment exercise. Scientometrics, vol. 74 (2), 309-316.

Thelwall, M. (2002a): A research and institutional size based model for national university web site interlinking, Journal of Documentation, vol. 58 (6), 683-694.

Thelwall, M. (2002b): Evidence for the existence of geographic trends in university web site interlinking. Journal of Documentation, vol. 58 (5), 563-574.

Thelwall, M. (2004): Link Analysis: An Information Science Approach. Academic Press; San Diego, p. 282

Thelwall, M., y Aguillo, I. F. (2003): La salud de las Web universitarias españolas. Revista Española De Documentación Científica, vol. 26 (3), 271-305.

Thelwall, M., y Harries, G. (2003): The Connection Between the Research of a University and Counts of Links to Its Web Pages: an Investigation Based Upon a Classification of the Relationships of Pages to the Research of the Host University. Journal of the American Society for Information Science and Technology, vol. 54 (7), 594-602.

Thelwall, M., y Harries, G. (2004): Do The Web Sites of Higher Rated Scholars Have Significantly More Online Impact? Journal of the American Society for Information Science and Technology, vol. 55(2), 149-159.

Thelwall, M.; Tang, R., y Price, L. (2003): Linguistic Patterns of Academic Web Use in Western Europe. Scientometrics, vol. 56 (3), 417-432.

Thelwall, M., y Zuccala, A. (2008): A University-Centred European Union Link Analysis. Scientometrics, vol. 75 (3), 407-420. 
Tolosa, G.; Bordignon, F.; Baeza-Yates, R., y Castillo, C. (2007): Characterization of the Argentinian Web, Cybermetrics, vol. 11(1), paper 3. http://www.cindoc.csic.es/cybermetrics/articles/v11i1p3.html [Consulta: 29-10-2008].

Vanti, N. (2005): Os links e os estudos webométricos. Ciência da Informação, vol. 34 (1), 78-88.

Vaughan, L. (2006): Visualizing linguistic and cultural differences using Web co-link data. Journal of the American Society for Information Science and Technology, vol. 57 (9), 1178-1193.

Vaughan, L., y Thelwall, M. (2005): A modeling approach to uncover hyperlink patterns: The case of Canadian universities. Information Processing \& Management, vol. 41 (2), 347-359. 